\title{
ACTUALIDADES
}

Rev. Chil. Pediatr. 62 (1); 61-68, 1991

\section{Nuevos conceptos acerca del síndrome hemolítico urémico}

\author{
Rodrigo Urízar E. ${ }^{1}$; Jorge Cerda $P^{1}{ }^{1}$; Ricardo Muñoz A. ${ }^{1}$; \\ Jill Largent $\mathbf{A}^{2}{ }^{2}$; Carlos Saieh $\mathbf{A}^{3}$
}

Hemolityc uremic syndrome

\begin{abstract}
According to the heterogenous nature of hemolitye uremic syndrome in relation to the etiology, pathophisiology, treatmente and diagnosis, we wich to draw attention to the main characteristics about its epidemiological clinical and immunopathoiogical asfects. The HUS's distributes through all the world, but in Argentina, North of Europe, South Africa and west of USA the incidence is higher than the rest of the countrjes. The :mmunopathological studies shows thrombotic angiopatic lesion, consisting ir generalized alteration of the capilar and arteriolar epithelium. Decreased levels of $\mathrm{PGi}_{2}$, Von Willebrand's factor and bacterial toxins are apparently involved among iriechanism that are able to produce HUS. Dialisis is one of the main helps in the treatment of HUS, and in spite of our cont:nued advances in knowledge about this disease, still Lurther developments a:e needed in pathophisiol. ogv and therapeutics to enlight its int:mate mechanisms.

(Kev vords: haemolityc uremic svondrome).
\end{abstract}

E1 sindrome hemolítico urémico (SHU) resulta de la acción de numerosos factores etiológicos y patogénicos. En la década del 50 Gasser $y$ col. sistematizaron la información existente sobre este problema y acuñaron el término de $\mathrm{SHU}^{1-8}$.

La información reciente ha aclarado considerablemente la naturaleza del cuadro. En la actualidad se observa aumento de su incidencia $y$ expansion a riveles etarios de adolescentes, adultos $y$ ancianos ${ }^{9-11}$, por to tanto, el pediatra, el internista y el geriatra deben familiarizarse con el SHU. Este escrito enfoca algunos aspectos etiológicos, patogénicos y terapéuticos de relevancia clínica práctica del síndrome, cuyas consecuencias pueden ser devastadoras. El tema ha sido evaluado en forma exhaustiva en otras publicaciones $2,6,12,17,5$ ?

\section{Características clínicas y epidemiológicas}

El SHU puede ocunir a cualquiera edad, es precedido generalmente por infección respira.

1. Albany Medical College. Internal Medicine, Nephrolagy Albany, New York, USA.

2. Albany Mcdical College. Pediatrics, Nephrology Albany, New York, USA.

3. Hospital Lujs Calvo Mackenna y Clínica Las Condes, Santiago, Chile. toria $\mathrm{o}$, más comúnmente, digestiva con diarrea. Los pacientes muestran palidez muy acentuada. la mayoría tiene hipertensión arterial, oligura, proteinuria, kucocituria $y$ hematuria, pudiendo haber hiperbilirrubinemia y alteraciones hidroelectroliticas.

El estudio hematológico revela anemia hemolitica microangiopática, poiquilocitosis, leucocitosis $y$ altos niveles sanguíneos de dehidrogenasa lactica, que se correlacionar en forma estrecha con la hemolisis y constituyen un indice sensible de actividad del síndrome. Las elevaciones de la urea y de la creatinina sérica reflejan el grado de compromiso de la función renal.

El daño del tiñón y de otros tejidos determi. nan la intensidad de las manifestaciones clínicas: oliguria prolongada, hipertensión arterial mantenida y refractaria, encefalopatia o enterocolitis severa ${ }^{2,6,13}$.

La distribución del SHU es mundial, con alta incidencia y prevalencia en Argentina, norte de Europa. Sudáfrica $y$ el oeste de los Estados Unidos $^{3}$ 14. 23. En esta última zona el promedio anual es de 0.41 a $1,16 / 10^{5}$ casos en niños neenores de 16 años de edad. Una incidencia más alta, 3,02/10 se observa en niños de 3 años 0 menos. Brotes epidémicos, con incidencja de $11,2 / 10^{5}$ ocurren frecuentemente en EE.LU. en nir̄os de 14 años. El SHU es endémico en algunas zonas metropolitanas del oeste de EE.UU., como Califomia, Oregón o Washington. Sin em. 
bargo, aparece en miniepidemias en todo el territorio de EE.UU. y Canadá ${ }^{24}$, 25 .

En Inglaterra, en los afos 1983 y 1984, se reportaron 31 y 46 pacientes con SHU, respectivamente, con una incidencia promedio anual de 0,25 a $0,36 / 10^{5}$ en niños menores de 16 años ${ }^{15}$.

En Chile no existen datos estadísticos de la real magnitud del SHU, a pesar de que la primera publicación sobre la materia se hizo en $1962^{16}$. Desde entonces han aparecido esporádicamente algunos brotes, pero sin poder catalogar ninguna zona como endémica ${ }^{17}$.

En general, se estima que aproximadamente 8\% de los pacientes con diarrea epidémica desarrollan $\mathrm{SHU}^{27}$. Observaciones recientes indican aumento de la incidencia en senescentes infectados con colibacilo enterohemorrágico productor de verotoxina ${ }^{11}$. En la década del 70 se de. mostró con cierta frecuencia SHU en mujeres jóvenes que usaban estrógenos en alta concentración como contraceptivo ${ }^{30}$. En la tabla 1 se of rece una clasificación de los síndromes micro. angiopáticos segün diferentes autores ${ }^{3,12,16,}$ $18,20,27,29,31,39,46,47,53,54,57,58$.

La evolución progresiva de la microangiopa. tía que lleva a insuficiencia renal terminal, diálisis o trasplante, es infrecuente. La insuficiencia renal terminal en 20 a $25 \%$ de los casos, descrita en las décadas del 60 y $70^{7,14}$, es demasiado ajta comparada con frecuencias actuales. En EE.UU. requieron trasplante renal 3 a $5,8 \%$ de los niños con SHU y sólo $0,2 \%$ de los adultos $y$ senescentes, probablemente debido a la mayor gravedad del SHU en estos últimos ${ }^{29}$. En Japón la incidencia de insuficiencia renal terminal debida al SHU varía entre 0,6 y 1,8 por ciento ${ }^{29}$. Afortunadamente en niños la recuperación espontánea o inducida por el tratamiento se aproxima a 90\%. En Chile 7,6\% del total de los pacientes descritos fallecieron, aunque este procentaje es significativamente más bajo desde el aึo 1983 en adelante $(2,5 \%)$ y sólo $2,5 \%$ quedan en insuficiencia renal crónica ${ }^{17}$.

Debido a la malignidad del SHU hereditario o recurrente (varjedad autosómica y recesiva) (tablas 1 y 2) y a su desarrollo en pacientes trasplantados, la mortalidad puede fluctuar entre 68 y $100 \%{ }^{27}$. La morbimortalidad se debe, en general, a complicaciones frecuentes y dificiles de controlar, como daño difuso del sistema nervioso central, descompensación cardiovascu.
Tabla 1

Clasificación de los sindtomes microangjopáticos (síndrome hemolítico urémico y púrpura trombocitopénico trombocito)

Primario:

Idiopático o prototípico

Hereditario

Familiar

Recurrente

Secundario:

Infeccioso/Toxinas

Bacterias

Virus

Riquetsias

Microtatobiote

Toxinas (no bacterianas)

Veneno de serpiente (principalmente DIC)

Ciclo sporina

Mitomicina

Lradjación

Procesos inmunológicos

Rechazo agudo

Enfermedades autoinmunes

Lupus eritematoso diseminado

Escletoderma

Hormonas

Embarazo, Preeclampsia, Eclampsia

Contraceptivos de alta concentración estrogénica

Microtrauma

Mal funcionamiento de válvulas cardíacas artificiales

Hemangioma cavernoso giqante (Síndrome de Kassabach-Merrit)

Secundario a Trasplante de Médula Osea.

lar, isquemia intestinal, hipercatabolismo, anemia hemolítica prolongada, hipertensión refractaria al tratamiento, caquexia e infecciones.

\section{Inmunohistopatologfa}

La histopatologia renal varía desde lesiones glomeruloarteriolares isquémicas e inflamatorias mínimas, hasta glomenuloesclerosis difusa y ne. crosis cortical ${ }^{1,2,8,27,30}$.

La microangiopatía o angiopatía trombóti. $\mathrm{ca}^{33}$, consiste en una alteración estructural generalizada del endotelio arteriolar y capilar. Las células se hinchan y se separan de la membrana basal desarrollándose necrosis y fibrosis. El espacio subendotelial ensanchado, con el colágeno expuesto a la fibronectina $y$ a otras proteinas circulantes promueven agregamiento y 
trombosis plaquetaria ${ }^{1,2,8,30,31}$. E1 mesangio prolifera, se expande $y$, coasionalmente, sufre necrosis llegando a lisis de la matriz y de la mem. brana basal ${ }^{31,32}$. Los leucocitos polimorfos $y$ mononucleares se observan frecuentemente en estas zonas. Las lesiones inflamatorias agudas o glomerulonefriticas son generalmente minimas e infrecuentes; sin embargo, su ausencia podria deberse a que la mayoría de las muestras del tejido renal se obtiene en periodos tardíos de la enfermedad. En general, la microangiopatía puede afectar predominantemente los glomérulos, las arteriolas o ambos (tabla 2). La microscopia de inmunofluorescencia revela depósitos capilares, mesángicos y arteriolares de fjbrinógeno y fibrina, fibronectina, lámina y factor de von Willebrand ( $v W)$ en número significativo de pacientes ${ }^{3,8,33}$. Con menor frecuencia, en forma aislada o asociada a las anteriores, se observan depósitos de inmunoglobulinas G y $\mathrm{M}$ en acúmulos granulares, lineares y mix tos.

\section{Patogénesis}

La microangiopatía guarda estrecha relación con la disfunción endotelial documentada clí. nica y experimentalmente; se ha postulado que en los casos de SHU postinfeccioso y recurrente disminuiría o cesaria la sintesis de prostaciclina
(PGI $)_{2}$ o productos similares (tabla 2$)^{3}$, lo que se corregiria con el tratamiento o espontáneamente. Se ha postulado, además, que los pacientes con SHU carecen de un factor plasmá. tico que estimularía la producción de $\mathbf{P G I}_{2}$. Sin embargo, la administración de prostaciclina no disminuye la actividad del SHU, mientras que en ciertos casos la infusión de plasma corrige el defecto ${ }^{3,5}$. Estos resultados se correlacionan con el aislamiento de un compuesto plasmático altamente polar (peso molecular de 300 a 400 daltons) que mantiene la función o previene la alteración del sistema enzinático de la $\mathrm{PGI}_{2}$.

Recientemente se ha estudiado lia síntesis, aimacenamiento, procesamiento y secreción del factor de $\mathrm{vW}$ en pacientes con $\mathrm{SHU}^{5}$. La producción de este elemento procoagulante por las células andoteliales, megacariocitos y plaquetas es modulada por genes ubicados en el cromosoma $12^{5}$. Los monómetros sintetjzados tienen pesos moleculares de 220 a 260 dalton, se glicosilan, dimerizan y polimerizan antes de ser excretados. Multímeros con peso molecular de 220.000 a $220.000 \mathrm{x}$ n permanecen almacenados en las células, detectándose en el plasma de pacientes en fase inactiva del SHU recurrente o del púrpura trombocitopénico trombótico (PTT); ellos desaparecer. con la activación de la microangiopatía y la trombocitopenia, secuencia que se invierte cuando la actividad cesa.

Tabla 2

Formas clúnicas del síndrome hemolítico urénico

\begin{tabular}{|c|c|c|c|c|c|c|}
\hline Tipo & $\begin{array}{l}\text { Agente } \\
\text { infeccioso }\end{array}$ & Pródromo & Hipertensión & $\mathrm{PGl}_{2}^{*}$ & Microangiopatía & $\begin{array}{l}\text { Pronós- } \\
\text { tico }\end{array}$ \\
\hline Prototípico & Desconocido & Gastroenterocolitis & $\begin{array}{l}\text { Intensidad va- } \\
\text { riable }\end{array}$ & Normal & $\begin{array}{l}\text { Predominantemente } \\
\text { glomerular }\end{array}$ & Benigno \\
\hline $\begin{array}{l}\text { Postinfec- } \\
\text { cioso }\end{array}$ & $\begin{array}{l}\text { E. coli } \\
\text { (0157:H7) } \\
\text { Shigella, } \\
\text { Salmonella, } \\
\text { Campilobacter, } \\
\text { Pneumococo, } \\
\text { Virus, etc. }\end{array}$ & Gastroenterocolitis & $\begin{array}{l}\text { Intensidad va- } \\
\text { rjable }\end{array}$ & $\begin{array}{l}\text { Dismi- } \\
\text { nuida }\end{array}$ & $\begin{array}{l}\text { Predominentemente } \\
\text { glomerular }\end{array}$ & Benigno \\
\hline $\begin{array}{l}\text { Asociado a } \\
\text { enfermedades } \\
\text { sistémicas }\end{array}$ & $\begin{array}{l}\text { Enfermedad pri- } \\
\text { maria }\end{array}$ & Enfermedad primaria & Severa & $\begin{array}{l}\text { Dismi } \\
\text { nuida } \\
\text { (puede } \\
\text { ser nor- } \\
\text { mal) }\end{array}$ & $\begin{array}{l}\text { Glomerular y arte- } \\
\text { riolar }\end{array}$ & $\begin{array}{l}\text { Enfer- } \\
\text { medad } \\
\text { primaria }\end{array}$ \\
\hline $\begin{array}{l}\text { Hereditario } \\
\text { securrente }\end{array}$ & Desconocido & Infección sistémica & Severa & $\begin{array}{l}\text { Dismi- } \\
\text { da }\end{array}$ & $\begin{array}{l}\text { Predominantemente } \\
\text { arteriolar }\end{array}$ & $\begin{array}{l}\text { Grave } \\
\text { recu- } \\
\text { rrente }\end{array}$ \\
\hline
\end{tabular}

* La concentración de PGI, medida en endotelio capilar-arteriolar. 
Posiblemente en el SHU (y en el PTT) se produ. ciría daño tóxico isquemico endotelial directo con liberación de multímeros (vW) de alto peso molecular. Estos, con el fibrinógeno y la fibronectina se acoplarian a las plaquetas, produciendo acúmulos y trombos en la pared capilar, cuya permeabilidad se altera significativamente ${ }^{5}$. Otras proteínas, como la lámina y la fibronectina se incorporan a los microtrombos y se depositan localmente, con lo que disminuye su concentración plasmática ${ }^{39}$. E1 factor de $v W$. la membrana plasmática de las células endoteliales y las membranas basales en general, poseen una capa monomolecular polianiónica glicosilada que repele elementos de carga negativa (impedancia electrónica). Es posible que la verotoxina $y$ la neuroaminidasa pneuftocúcica remuevan el polianion y generen o faciliten el desarrollo de lesiones inflamatorias, isquémicas $y$ necróticas $5,40,41$. E1 transporte de las toxinas por proteínas plasmáticas y su captación ulterior por receptores celulares constituiria otro posible mecanismo de daño tóxico.

La neutralización de los compuestos gicosilados del factor de wW permitiria la aglutinación plaquetaria directa, sin que intervengan otros mediadores ${ }^{5}$. Presumiblemente la toxina enterohemorrágica de la $E$. coll $0157: \mathrm{H} 7$ produciría inicialmente aumento de la sintesis de $\mathrm{PGl}_{2}$ seguida de su desaparición ${ }^{3,42,43}$. La inoculación de la toxina en conejos produce alteraciones histopatológicas y funcionales endoteliales semejantes a las observadas en el SHU ${ }^{44}$; además, la toxina induce cambios importantes en cultivos de células endoteliales humanas ${ }^{45}$. $\mathrm{La}$ neuraminidasa pneumocócica, cuya estructura química es similar a la de la verotoxina, remueve la capa glicosilada externa exponiendo el antígeno de Thomsen-Friedenreich, un receptor superficial rico en ácido neuraminico presente en las células endoteliales glomerulares, eritrocitos y plaquetas ${ }^{46}$. Estos hallazgos se han documentado en casos de SHU o de sindromes semejantes producidos por infección preumococica, en los que, a diferencia del SHU clásico, se observa septicemia, coagulación intravascular diseminada y anemia hemolítica con Coombs positivo ${ }^{4,46}$. Las toxinas de Shigella disenteriae 1, del Streptococcus pneumoniae y Campylobacter jejuni, aislados de algunos casos de SHU inducen diarrea sanguinolenta y contienen verotoxina ${ }^{10,11,49,57}$. En una publicación re. ciente, 60\% de los pacientes evidenciaron infec- ción con E. coll 0157:H7, productor de verotoxinas; $15 \%$ tenian serología positiva, mientras que en $75 \%$ el cultivo y la serología eran positi. $\operatorname{vos}^{50}$. En un brote de diarrea producido por colibacito 0157:H7 se documentó colitis hernorrágica en 73 individuos de un hogar de ancianos; 12 personas $(16,4 \%)$ desarrollaron SHU, de los cuales [ $1(91,6 \%)$ fallecieron. No se observaron casos de SHU entre los empleados de dicha institución $^{11}$. Esta nueva variedad prototípica de colibacilo, descubierta en 1982, en casos de diarrea epidémica ${ }^{52}$ produce verotoxinas diferentes de las enterotoxinas termoestables o termolábiles elaboradas por colibacilos de otros serotipos. Las verotoxinas, cuyo nombre se debe a su toxicidad específica para líneas de cultivo de células Vero (o Hela), que son neutralizadas por anticuerpos contra la toxina de Shigella disenteriae $I$, se han denominado Verotoxina 1. Aquellas no neutraizzables por dicho anticuerpo. constituyen la Verotoxina 2 12, 57. Estas bloquean la reabsorción de liquido intestinal, al contrario de la toxina del bacilo del cólera, que produce un exceso de secreción por la mucasa enteral. La sintesis de verotoxinas es modulada por bacteriofagos, que al infectar el microorganismo activan los genes productores ${ }^{12,57}$. Las verotoxinas interfieren con la sintesis proteica causando eventuslmente la muerte celular. Debido a que la asotiación de las infecciones con Shigella disenteriae y la $E$. coli enterohemorragica con el SHU ha sido documentada, es posible que sus mecanismos patogénicos sean semejantes. El impacto que estas bacterias tienen en la salud pública es altamente significativo, ya que no sólo producen diarrea infecciosa to complicada y colitis hemorrágica, sino que tambièn SHU $y$ $\mathrm{PTT}^{12,19,49,57}$. Por lo tanto, la $E$. coll enterohemorrágica, la Shigella disenteriae 1 , el $S$. pneumoniae, el Campvlobacter jejuni y otros como Saimonellae. Hemophilus influenzae y Yersinia, podrian considerarse agentes establecidos del SHU y PTT, a traves de mecanismos patogenicos relativamente conocidos, de los que existen numerosas incognitas. Otros factores también pueden producir daño endotelial difuso y destrucción de las membranas plasmáticas con desarrollo de microangiopatía (tabla 2) ${ }^{3}, 58,59$.

Investigaciones recientes indican que la activación de los leucocitos polimorfonucleares y la formación de radicales de oxigeno ocurre en periodos tempranos del síndrome. Este mecanismo patogénico hasta ahora ha sido poco explorado, 
a pesar de que contribuye a producir alteraciones histopatológicas ${ }^{60,51}$. Además es muy probable que el ataque directo a la membrana celular de los glóbulos rojos resulte en hemólisis y que sea esta alteración de la membrana celular el mecanismo principal de la anemia hemolitica en lugar del atrapamiento y destrucción de los eritrocitos por mallas de fibrina, que raramente se observan en el SHU.

A estas alturas el lector se habrá percatado deI paralelo establecido entre SHL y PTT, $y$, por lo tanto, que los conceptos patogénicos acerca del primero, discutidos aqui, son aplicables también a este último ${ }^{5,62,63}$ En realidad, debjdo a su estrecha relación, estos dos síndromes deberian ser considerados variacjones de un proceso básico, prototipo de las microangiopatias ${ }^{3}$. En la tabla 3 se comparan algunas caracteristicas de ambos.

\section{Diagnóstico}

La detección precoz del SHU requjere que el médico piense en este trastorno $y$ realice algunos exámenes de laboratorio, como de orina completo, hemograma, recuento de plaquetas, electrolitos, urea y creatinina sérica, particularmente en casos de diarrea persistente o sanguinolenta. Los cultivos de deposiciones destinados a identificar la $E$. coli $0157: \mathrm{H} 7$ son indispensables. Sin embargo, en la mayoría de los hospitales no se practica este examen, debjdo a falta de antisueros adecuados. disponjbles sólo en institucio- nes especializadas ${ }^{54}$. La $E$. coll es denostrable en las deposiciones dos dias después de iniciada la diarrea, hasta aproximadamente dos semanas ${ }^{50,57,64}$. En la actualidad la identificación rápida de la $E$. coli $0157:$ H7 se basa en la incapacidad de esta bacteria para fermentar el sorbitol incluido en la placa de agar de McCon. $k^{5 e y^{50,}}{ }^{64}$, que más de $90 \%$ de los demás serotipos del colibacilo fermentan ${ }^{64}$. Como el procedimiento detecta solamente el serotipo $0157: \mathrm{H} 7$, otros estudios deben llevarse a cabo para una identificación más precisa de las $E$. coli enterohemorrágicas $^{12}$. La muestra, si no es cultivada inmediatamente, debe mantenerse a $-70^{\circ} \mathrm{C}$, para su estudio posterior y envío a otras instituciones ${ }^{54}$.

Un hallazgo de gran importancia práctica demuestra que la verotoxina acompaña a la gran mayoria de las $E$. coll $0157: \mathrm{H} 7$ aisladas de las deposiciones de pacientes con SHU. Se ha su. gerido que la jdentificación de la toxina en estas condiciones constituye la evidencia clínica confirmatoria más importante de infección con la variedad enterohemorrágica $0157: \mathbf{H} 7^{52}$. Es posible que usando anticuerpos monoclonales, la jdentificación de la verotoxina en la muestra de deposiciones pueda realizarse en forma más rápida $y$ eficiente $e^{57}$. Más aún, pacientes con alto riesgo de desarollar SHU pudieran ser identificados precozmente y tratados con antibióticos apropiados 0 inmunizados con antitoxina (globulina hiperinmune) o con "verotoxoide" $" 12,50,58$.

Tabla 3

Comparación de algunos clementos clínicos y de laboratorio en el sindrome hemolítico urémico (SHU) y el púrpura trombocitopénico trombótico (PTT)

\begin{tabular}{|c|c|c|}
\hline MANIF FSTACIONES CLINICAS & $\begin{array}{l}\text { SHU } \\
\text { (Von Gasser, 1955) }\end{array}$ & $\begin{array}{l}\text { PTT } \\
\text { (Moschcowitz, 1925) }\end{array}$ \\
\hline $\begin{array}{l}\text { Edad } \\
\text { Anemia Hemolítica }\end{array}$ & $\begin{array}{l}\text { Predomina en nij̄os } \\
\text { En todos, mínirna a } \\
\text { severa }\end{array}$ & $\begin{array}{l}\text { Predomina en adultos } \\
\text { En todos, grave }\end{array}$ \\
\hline $\begin{array}{l}\text { Trombocitopenia } \\
\text { Insuficiencia renat } \\
\text { Hipertensión arteriat } \\
\text { Encefalopatia } \\
\text { Haptoglobina } \\
\text { LDH } \\
\text { Coombs } \\
\text { G-6PD, Glutatión } \\
\text { Hematuria/proteinuria } \\
\text { Cilindros Hemáticos } \\
\text { Frotis Periférico }\end{array}$ & $\begin{array}{l}\text { En la mayoria } \\
\text { En todos } \\
\text { Frecuente } \\
\text { Frecuente } \\
\text { Disminuida } \\
\text { Aumentada } \\
\text { Nomal } \\
\text { Normal } \\
\text { En todos } \\
\text { Frecuente } \\
\text { Poiquilocitosis } \\
\text { (Esquistocitos) }\end{array}$ & $\begin{array}{l}\text { En todos } \\
\text { Infrecuente } \\
\text { Infrecuente } \\
\text { En la mayoría } \\
\text { Disminuida } \\
\text { Aumentada } \\
\text { Normal } \\
\text { Normal } \\
\text { Con compromiso renal } \\
\text { Con compromiso renal } \\
\text { Poiquilocitosis } \\
\text { (Esquistocitos) }\end{array}$ \\
\hline
\end{tabular}




\section{Tratamiento}

En Jos años 1960 y posteriores se demostró que la diálisis precoz de los pacientes con SHU aumentaba significativamente la sobrevi$\mathrm{da}^{7,8}, 17,24$, estableciéndose este procedimiento como su terapia fundamental. La simplicidad técnica, la disponibilidad en la mayoría de los hospitales y la buena tolerancia de los pacientes hacen la diâlisis peritoneal el tratamiento preferido, especialmente en los niños menores, con excepción de aquellos con estados hipercatabólicos avanzados o lesiones intestinales necróticas extensas.

Los casos de intensidad minima requieren sólo tratamiento médico general y balance hidroelectrolitico adecuado, anticipándose a las complicaciones cardiovasculares y neurológicas que pueden ocurrir inesperadamente. De acuerdo con la fisiopatología descrita antes, el uso de plasmaféresis, recambio o infusiones de plasma y/o de sangre estarían plenamente indicados, en especial en pacientes con lesiones predominantemente arteriolares. Estas medidas terapéuticas interrumpirían la continuidad del proceso patogénico del $\mathrm{SHU}^{3,5,8,65,68}$. Sin embargo, la plasmaféresis requiere equipos caros, entrenamiento del personal y presenta dificultades en niños menores, ${ }^{86}$. Las infusiones de plastna aparentemente producen resultados similares, aunque su eficacia no está del todo probada ${ }^{69}$, 70. Estas deben ser realizadas bajo estricto control médico, utilizando volúmenes de 5 a $10 \mathrm{ml} \cdot \mathrm{kg}$, sólo en pacientes sin sobrecarga o descompensa. ción cardiovascular.

En los casos de hipertensión arterial e insuficiencia cardíaca congestiva o edema pulmonar, la hipervolemia debe ser resuelta (diálisis, diuré. ticos o ambos) antes de proceder a la infusión de plasma. Las transfusiones de plaquetas deben usarse sólo en pacientes con recuentos plaquetarios muy bajos (menos de 20.000) o con sangramiento activo.

El trasplante renal en el SHU es el tratamiento de elección en los pacientes con insuficiencia renal terminaj. EJ SHU puede recurrir en pacien. tes trasplantados con riñón donado por un pariente. Las recurrencias se observan con menor frecuencia cuando se utiliza riñón de cadá$\operatorname{ver}^{73}$. Además, la reacción de rechazo agudo, la toxicidad de la ciclosporina A y de la globulina antilinfocítica y la hipertensión arterial producen daño endotelial semejante a los del SHU original, complicando considerablemente la intervención de estos hallazgos ${ }^{71,73}$. En una serie de 14 pacientes sometidos a trasplante, la recurrencia del SHU fue documentada en 7 y otros 3 mostraron microangiopatía sin enfermedad clínica, a raíz de lo cual se ha recomendado eva. luaciones éticas y clínicas muy precisas en pacientes propuestos para recibir un riñón donado por un pariente inmediato, pues es posible que se transmitan factores que favorecen la recurrencia del SHU, to que tendría mayor trascendencia clínica que la posible disminución de las reacciones de rechazo ${ }^{77}$. Sin embargo, estos hechos nо han sido comprobados por otros centros, y los trasplantes renales en el SHU continúan sin que aumente la morbimortalidađ atribuible a la recurrencia del sindrome. Para prevenir este fenómeno o el desarrollo de cuadros clínicos semejantes al SHU, se han hecho modificaciones al tratamiento inmunosupresivo, excluyendo de él la ciclosporina A y la inmunoglobulina antilinfocítica, agregándole aspirina y dipiridamol. La observación prolongada previa al trasplante, debería considerarse requisito clínico indispensable; sin embargo, no han podido ser establecidas la duración de ésta $y$ la conducta a seguir en casos de recurrencia del SHU antes o después del trasplante.

A pesar de adclantos notables en su conocimiento, aún no se definen con certeza los mecanismos del SHU. La identificación bacteriana rápida y precoz, las posibilidades de tratamiento específico o inmunización de los pacientes, son objetivos que pudjeran ser de gran utilidad práctica en la prevención y curación de este síndrome. Por último, la idea de Remuzzi y $\mathrm{col}^{74}$ de crear un registro intemacional para infonmación estándar acerça del SHU, es de extriordinaria trascendencia clinica. Es de esperar que cristalice en estudios multicéntricos prospectivos.

\section{Resumen}

E1 síndrome hemolítico uremico (SHU) es heterogéneo en su etiologia, fisiopatologia, tratamiento y diagnóstico. En esta presentación se analizan algunos aspectos de su epidemiọlogía, clínica e inmunopatología. La distribución del SHU es universal, pero en Argentina, sur de Europa, Sudáfrica y oeste de USA se detecta con mayor frecuencia que en el resto de los países. Los estudios inmunopatológicos 
muestran lesiones angiopáticas trombóticas consistentes en alteración generalizada del epitelio capilar y arteriolar. Entre los factores que aparentemente participan en la genesis del sindrome se analizan la disminución de los niveles de prostaglandina $\mathrm{PGT}_{2}$, del factor de von Willebrand y las toxinas de origen microbiano. La diálisis es una de las herramientas más útiles en el manejo del SHU. A pesar de los actuales conocimientos, todavia se requiere más unvestigación para conocer los mecanismos intimos del síndrome.

(Palabras clave: síndrome hemolítico urémico.)

\section{Referencias}

1. Gasser, C.; Gautier, E.; Stek, A.; Siebenmonth, E.E.; Oechslin, $R$.: Haemolytich Uremische: BiJaterale Nierendindennekrosen Bei Akuten Erworbenen Haemolytschen. Schweiz Med Woschenschr 1555; 85: 905-909.

2. Fong, J.S.; De Chevadarian, Y.P.; Kaplan, B.S.: HUS. Current concepts and management. Pediatr Clis North Am 1982; 29: 835-856.

3. Remuzzi, G.: HUS and TTP: variable expression of a single entity. Kidney Int 1987; 37: 292-308.

4. Scully, R.; Mork, E.J.; McNeely, B.: Case records of the Massachusetts General Hospital. Case 151986. N Engl J Med 1986; 314: 1032-1040.

5. Byrnes, J.J.; Mooke, J.L.: TTP and HUS syndrome; envolving concepts of pathogenesis and therapy. Clin Hematol $1986 ; 15 ; 413-442$.

6. Kaplan, B.S.; Thompson, P.D.; Chodavarion, J.P.: The HUS. Pediatr Clin North Am 1976; $23: 761$ 777.

7. Gianantonio, C.; Vitacco, $M_{. ;}$Mendilahara. $F_{\text {; }}$ Gollo, G.E; Solo, E.T.: The HUS. Nephron 1973; $11: 174 \cdot 192$.

8. Urizar, R.E.: HUS. In Pediatric Nephrology. New Directions in Therapy. Ed. Urizar, R.E. Largent, J.; Gilboa, N. Med Exanl Publishing. Co. New Hyde Park, N,Y, 1983, pp. 176-216.

9. Clarkson, A.R.; Lawrence, J.R.; Meadows, R. et al.: The HUS in adults, Q. J Med 1970;39: 227244.

10. Netll, M.A.; Agöti, J.: Rosen, $H_{*}$ : Hemorrhagic colitis with $E$. coli $0157: \mathrm{HT}$ preciding adult HLS. Arch Int Med 1985; $145: 2215-2217$.

11. Carter, A.O.; Borczyk, A.A.: Corlson, J.A.K.; Harvey, B.; Hockin, J.C.; Karmaly, M.A. et al.: A severe outbreak of $E$. coll $0157: \mathrm{H} 7$ associated with hemorrhagic colitis in a nursing home. N Engl J Med 1987; 317: 1496-1500.

12. Cleary, T.G.: Cytotoxin producing $E$. coll and the HUS. Pediatr Clin North An 1988; $35: 485-501$.

13. Shet, K.J.; Swick, $H_{\text {; }}$, Howorth. $\boldsymbol{N}_{-}$: Neurologyc involvement in HUS. Ann Neurol 1986; 19: 9093.

14. Gianantonio, C.; Vitacco, M.; Mendilaharzu, $F$. ; Sojo, E, et al.: The HUS. J Pediatr 1964; 64: 478-491.
15. British Pediatric Association. Communicable Disease Survillance Centre; Survillance of HUS, 1983, 4. Br Med J 1986;292: 115-117.

16. Vildósola, $f_{\text {; }}$ Bravo, $M$,; Emparanza, E.: Síndrome Hemolítico Urémico en la Infancia. Pediatría (Santiago) 1962;5:292-309.

17. Cordero, J.: Fielbaum, O.; Varela, M.; Saieh, C.; Baeza, J.: Síndrome Hemolítico Utémico; experiencia de 154 casos. Rev Chil Pediatr 1990; 61: 235-242.

18. Mothies, H.; Lecler, F.; Habib, R.; Royer, P.: Etude clinique et biologique de 37 observations de Syndrome Hemolitique et Uremique. Arch Fr Pediatrie 1969;26:369-390.

19. Trompeter, R,S, Schwartz, $R$.; Chanter, C. et al.: HUS: analysis of prognosis features. Arch Dis Child 1983;58: 101-105.

20. Donckerwolcke, R.A.; Kü̈ten, R.H.i Tiddens, H.A. et al.: HUS. Paediatrician 1979; 8; 378 393.

21. Kibel, M.A.; Barnard, P.J.: The HUS: a survey in southern Africa. S Afr Med J 1968; 42: 692-698.

22. Lieberman, E.: HUS. J Pediatr $1972 ; 80: 1-6$.

23. Sorrenti, L.Y.; Lewy, P.R.. The HUS. Am J Dis Child 1978; 132: $59-62$.

24. Tune, B.M.; Leavit, T.J.; Grieble, T.J.: The HUS in California; a review of 28 non heparinized cases with long term folow up. J Pediatr 1973; 82: 304-310.

25. Brasher, C.: Siegler, R.L. The HUS, J Med 1981 134: 193-197.

26. Rogers, M.F.; Budnick, L.D.; Kirson, I. ef al. HUS, an outbreak in Sacramento, Catiformia. J Med 1986; 144: 166-173.

27. Torn, P.I.; Hickmon, R.O.: HUS a population based study in King County, Washington, 1971, 80. Pediatrics 1987;80:41-45.

28. McLean, M.M.; Jones, C.H.; Southerland, D.A. HUS; a report of an outbreak. Arch Dis Child $1966 ; 41: 74-81$.

29. Kaplan, B.S.: Observaciones no publicadas.

30. Brown, C.B.; Clarkson, A.R.; Robson, J.S. et al: HUS in women taking oral contraceptives. Lancet 1973 ; 1 : 1479-1481.

31. Herbert, D.: Observaciones no publicadas.

32. Habib, R.; Mathieu, H.; Royer, P.: Le syndrome hemolitique et uremique de l'enfant. Nephron $1967 ; 4 ; 139-172$.

33. Shigematst, H.; Kikman, S.H. Churg J. et al. Mesangial involvement in HUS. A light and electronic microscopic study. Am J Pathol 1976; $85: 349-362$.

34. Goldstetn, M.H.; Churg, J.; Strouss, L.; Gribetz, D. - HUS. Nephron 1979;23: 263-272.

35. Takemura, T.; Yoshioka, $K$,; Myamoto, $H$. Matsumoto, K. et al.: Glomerular deposition of cross linked fibrin in human kidney diseases. Kidney Int 1987:32:102-111.

36. Berman, $W, J$.: The HLS, initial presentation mimicking ulcerative colitis. J Pediatr 1972;81: 277.287.

37. Peterson, R.B.; Meseroll, W.P.; Shrogo, G. et al. Radiographic features of colitis associated with the HUS. Radiology 1976, 118:667-669. 
38. Croner, G.E.: Burdick, G.E.: Acute colitjs resembling ufcerative colitis in the HUS. Digest Dis 1976; 21: $74-76$.

39. Schwatz, D.L.; Becker, J.M.: So, H.B. et al. Segmental colonic gangrena. A surgical emesgency in the HUS. Pediatrics $1978 ; 62: 54-56$.

40. Deckmyn, H, Zojo, C.; Arnout, J.: Partial isolation and function of the prostacyclin regulating plasma factor. Science 1985;69: 383-393.

41. Cerda, J.: Crizar, R.E.: Conran, R. et al.: Disseminated intravascular coagulation (DIC) induced by liquoid (polyanetholsulfonate) in the rat. Effect on circulation fibronectin. Br J Exp Path $1986 ; 67: 623-628$.

42. Cooke, R.P.D.: Rose, P.E.: Classification of the HUS. J Clin Path 1986; $39: 812-813$.

43. Rose, P.E.; Armour, J.A.; Williams, C.E. et al.. Verotoxin and neuraminidase induced platelet aggregating activity in plasma; their possible role in the patlogenesis of the HUS. I Clin Path 1985; $38: 438-441$.

44. Kent, R.S.; Dietrich, S.L.; Whorton, A.R.: Regulation of vascular prostaglandin synthesis by ntetabolites of arachidonic acd in pertused rabbit aorta. J Clin Invest 1983;72:455456.

45. Fong, J.S.C.; Kaplan, B.S.: Impairment of platelet aggregation in HLS: evidence for platelet exhaustion. Blood 1982;60: 564-567

46. Raif, L.; Keane, W.F.; Mchael, A.F.: Unilateral Shartzman reaction: corlical necrosis in one kidney following in vivo perfusion with endotoxir. Kidney lnt 1977;12:91-95.

47. Harlan, J.M.; Harcker, L.A.; Reidv, M.A.: Ljpopolysaccharje-mediated bovine endothelial cell injury in vitro. Lab Invest $1983 ; 48: 269-274$

48. Kein, P.J.; Bullo, M.: Newman, R.A.: Muller, P.: Ulenruck, $G$. et of.: Thomsen-Friedenrejch antjgen in HUS. Lancet 1977: 1: 1024-1025

49. Alon, U, Alder, S.P.; Chan, J.C.M.: HUS associated with streptocuccus pntumoniar. Ain J Dis Child $1984 ; 138: 496-499$

50. Feld, L.G,; Springate, J.E; Darragh, R.: Pneumo coccal pneumoniae and HUS. Pediatr Infect Dis $1987 ; 87: 248-250$.

51. Riley, L.W.; Remis, R.S.; Helgerson, S.D. et al. Hemorrhagic colitis associated with a rare $E$. coll serotype. N Engl J Med 1983; 308:681-685.

52. Karmali. M.A.; Perric, M.; Lim. C.: The association between idiopathic HUS and infection by verotoxin-producing $E$. coli $0157: \mathrm{H} 7$. J infect Dis $1985 ; 151: 775-782$.

53. Grandsen, W.R.i Damm, S.: Andersson, J.D.: Further evidence associating HUS with infection by verotoxin-producing $E$. coli 0157:H7. I Infect Dis $1986 ; 154: 522-523$

54. Ratnam. S.; March, S.; Ojoh, C.: HUS associated with verotoxin producing $E$. colt. Can Med Assoc $1985 ; 133 ; 37-38$

55. Martin, M.L.; Shipman, L.D.; Potter, M.E.; Was $x h$ smuth, I,K.; Wells, J.G.; Hedberg, K.; Tauxe. $R, V$ : lsolation of $E$. coli $0157: \mathrm{H}$ ? from dairy cattle associated with two cases of HUS. Lancet $1986 ; 2$ : 1043 .

56. HUS associated with $E$, coli $0157: H 7$. Enteric Infect USA 1984-MMWR 1985; 34: 20-21.
57, Koster, F.T.; l,elin, J.; Walker, L.: Tung, K.S.K.; Glisman, R.H.: HUS after shigellosis. Relation to endotoxinemia and circulating immune complexes. N Engl J Med 197B; 298: 927-933.

58. Koster, F.T.; Boonpucknatig. $V$.; Sujco, $S_{\text {.; }}$ Gilman, R.H.; Raham, M.M.: Renal histopathology in the HUS following shigellosis. Clin Nephrol $1984 ; 21: 126-133$

59. Sack, R.B.: Enterohemorrhagic E. coli. N Eng J Med 1987;317: 1535-153?.

60. Drummont, $K . N_{\text {: }}$ HUS, then and now. N Engl J Med 1985; 312:116-118.

61. Kaplan. B.S : Drummond, $\boldsymbol{K}$.: HUS is a syndrome. N Engl J Med 1978; 298: 964-966.

62. Bolande, R.P.: Kaplan, B.S.: Experimental studies on the HUS. Nephron $1985 ; 39: 228-236$.

63. Forsyth, K.D.; Sympson, A.C., Fïtzpatrick, M.M.: Neutropil-mediated endothelial injury in HLS. Lancet $1989 ; \mathrm{i}: 411-414$.

64. Ridolfi, R.I.; Bell, M.R.: Thrombotic thrombocytopenle purpura. Report of 25 cases and review of the literature. Medicine $1981 ; 60: 413-420$.

65. MacWinney, J.B.; Packer, I.T.; Miller, G. et al. Thrombotic thrombocytopenic purpura in children. Blood 1962;19:181-199.

66. Spika, J.S,; Parsons, J.E.; Nordenberg, D.: HUS and diatrhea associated with $E$. coli 0157:H7 in a day care centre. J Pediatr 1986; 109: 287. 291.

67. Misiant, R.; Apiani, A.C.; Edefonti, A. et al.; HLS: therapeutic effect of plasma infusion. Br Med J 1982; 285:1304-I306.

68. Taft, E.G.: Thrombotic thrombocytopenic purpura and dose of plasma exchange. Blood 19?9: 54: 842-849.

69. Rizzoni, G.; Claris-Appiani, A.; Edefanti. A. et al.: Plasma infusion for HLS in children: results of a multicenter controled trial. J Pediatr 1988; 112: 284-290.

70. Iit, E.T.; Linker, C.A.; Shuman, M.A.: Management of treatment failures in thrombotic thrombocytopenic purpura. Am J Hematol 1986; 23 : $347-361$.

71. Loirat, $C_{*}$ Sonsino, E.; Hinglais, $N$, (on behalf of the French Society of Pediatric Nephrology). Treatment of childhood HUS with plasma. A multicentre zandomized controlled trial. Pediatr Nephrol 1988; 2: 279-285.

72. Cole, B.R.: Plasma infusion therapy in HUS: is it unwarranted? Pediat Nephrol 1988; 2: 286287.

73. Hebert, D.: Sibley, R.; Mauer, S.M.: Recurrence of HUS in tenal transplant recipients. Kidney Int $1986 ; 30: \$-51-5-58$.

74. Zoja, C.; Furci, L.; Ghitardi, F.: Cyclosporineinduced endothelial injury, Lab Invest 1986; $55: 455-462$.

75. Cattell, $V_{\text {.: }}$ Mitomycin induced hUS. An Experimental model in the rat. Am $J$ Pathol $1985 ; 12$ : 88-95.

76. Renuzzi, G.: Zoja, C.; De Gretano, G.; Rossi E.C.: Prostacyclin and HUS: from the laboratory to and International registry. Int J Artif Organs $1987 ; 10: 337-340$. 